\title{
Radio Resource Allocation in D2D Underlay Communication Using Two Phased Auction Based Fair and Interference Resource Allocation
}

\author{
Alfiya Syifana $^{1}$, Linda Meylani ${ }^{2}$, V.S.W. Prabowo ${ }^{3}$ \\ ${ }^{1,2,3}$ School of Electrical Engineering, Telkom University, Bandung, 40287, Indonesia \\ 1,2,3alfiyasyfn@student., lindameylani@,vinsensiusvsw@telkomuniversity.ac.id \\ Manuscript received July 15, 2021; revised August 26, 2021; accepted October 7, 2021.
}

\begin{abstract}
The high demands of the mobile user will affect the workload of eNodeB, which results in the decreasing performance system of eNodeB. Device-to-Device (D2D) underlaying communication system is a solution in reducing the workload of eNodeB and increasing the system data rate. This communication system consists of two users, namely Cellular User Equipment (CUE) and D2D pair, where CUE shares its resources with the D2D pair. This sharing of resources also causes interference and should be managed using the resource allocation algorithm. This research used the TAFIRA D2D algorithm and compared it with the greedy algorithm and the TAFIRA CUE algorithm. The research calculates parameter performance of the system, such as spectral efficiency, power efficiency, and fairness among D2D pairs. The simulation results show that Greedy algorithm has a better performance compared with TAFIRA algorithm. TAFIRA D2D only can achieve $19.94 \mathrm{bps} / \mathrm{Hz}$ in spectral efficiency, $23.88 \mathrm{Kbps} /$ watt in power efficiency, and $89 \%$ fairness among D2D pairs.
\end{abstract}

Keywords: D2D; Underlay Communication; TAFIRA; Greedy; Resource Allocation

DOI: $10.25124 /$ jmecs.v8i2.3972

\section{Introduction}

New data-intensive applications are emerging in the daily routines of mobile users. At the same time, telecom operators are struggling to accommodate the high demands of mobile users [1]. The high demands of the mobile user will affect the workload of eNodeB, which results in the decreasing performance system of eNodeB. The D2D communication is direct communication between the device to device without going through eNodeB. Thus Device to Device (D2D) communication can mitigate the load of eNodeB while also increasing the system capacity [2].

Underlay D2D communication uses the same uplink or downlink channel or both channels of Cellular User Equipment (CUE) simultaneously[3]. This communication affects the increase of spectral efficiency and power efficiency [4]. However, it also affects the interference between users inside the system that interrupts the communication with the device [5]. In particular, if the management of interference D2D communication is not properly enough, it severely degrade the performance of the cellular network [6].

Work [7] proposed a novel joint power control and resource scheduling scheme to enhance both the network throughput and the users fairness of the underlay D2D communication networks. Work [8] compared a two-phase auction-based, fair, and interference aware resource allocation algorithm (TAFIRA) with a minimum knapsack-based interference resource allocation algorithm (MIKIRA) and obtains a much better system sum-rate when compared with MIKIRA. Work [9] proposed a new algorithm based on a local search algorithm and compared it with two existing algorithms (MIKIRA and TAFIRA).

This research discusses the allocation of the resource block (RB) using the TAFIRA D2D algorithm to decrease the interference and also to obtain a better performance parameter. This algorithm allocates 


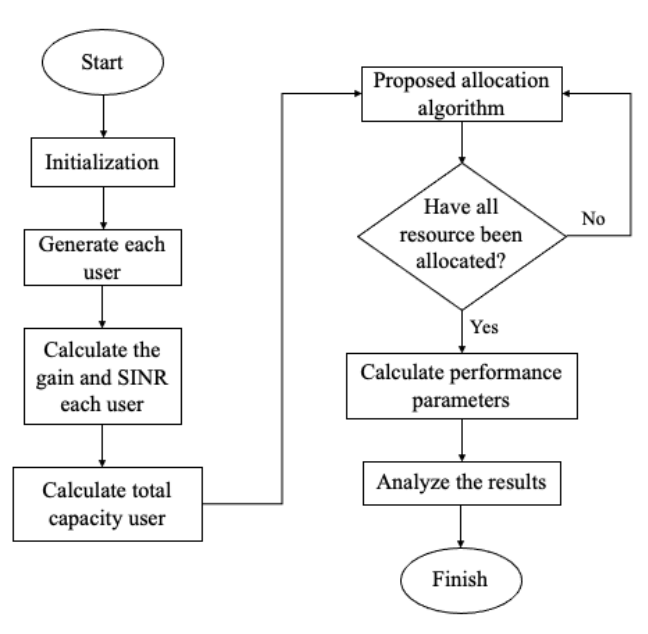

Fig. 1. Simulation Flow

the resource based on capacity of the D2D pairs and the CUE side. In this simulation, the D2D pairs are varied. After that, TAFIRA D2D compares with TAFIRA CUE and Greedy algorithm to analyze the performances. The simulation shows that the TAFIRA algorithm cannot improve the system performance.

\section{Simulation Process}

Figure 1 shows the simulation flow of this research. This research starts with initialization and generates user. The path loss value got from the calculation of distance between users. Then the gain channel, Signal to Noise Interference (SINR) value, and capacity of each user are calculated. After that, the proposed allocation algorithm allocates all of the CUE's resources to the D2D pair. Then the performance parameters are calculated and analyzed.

\subsection{System Model}

The system model is a single cell to avoid interference from other users in other cells, the user is idle to keep away from the handover occurred. The cell contains an eNodeB, D2D transmitter (D2D Tx), D2D Receiver (D2D Rx), and CUE. This research focuses on uplink communication. CUE should be more than D2D pair because D2D pair need CUEs resources to communicates in the system. Each D2D pair is only allowed to be paired with a CUE. Utilization of resources simultaneously by CUE and D2D pair affects the interference. The signal from D2D Tx affects the interference to eNodeB, and the signal from CUE affects the interference to D2D Rx. Figure 2 explain about the utilization of resource simultaneously by CUE with D2D and interference received by users.

Equation 1 shows Urban Micro Systems (UMi) to calculate the path loss on the distance of users [10].

$$
P L_{i, j}=36.7 \log _{10}(d)+22.7+26 \log _{10}(f c)
$$

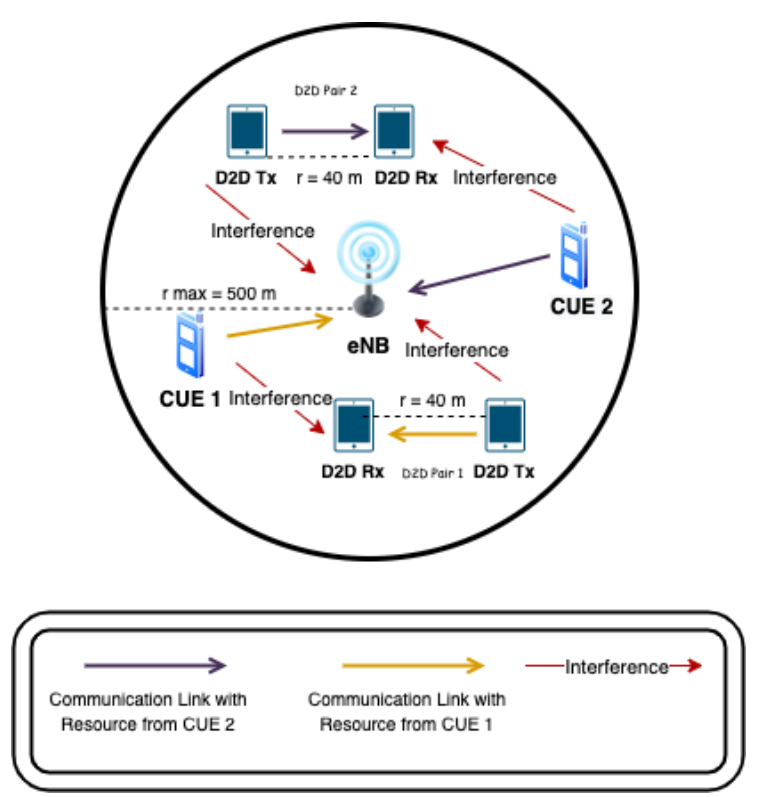

Fig. 2. System Model

where $\mathrm{d}$ represents a distance in meter, and $\mathrm{fc}$ represents the working frequency in the system in gigahertz. Pathloss in Equation 1 is used to find the gain of a channel using Equation 2 [11].

$$
G_{i, j}=P L_{i, j}+X_{\sigma}+\prod
$$

where $P L_{i, j}$ represents the path loss value in $\mathrm{dB}, X_{\sigma}$ represents the large scale fading with Gaussian distribution, and $\prod$ represents the small scale fading with Rayleigh distribution.

SINR is a comparison of the main signal with the interference and noise values that arise [12]. Equation 3 calculates the SINR value of eNodeB, whereas Equation 4 calculates the SINR value of D2D.

$$
\begin{gathered}
\gamma_{i, j}=\frac{P_{i} \cdot G_{i, e N B}}{N_{o}+P_{j} \cdot G_{j T x, e N B}} \\
\beta_{j T x, j R x}=\frac{P_{j} \cdot G_{j T x, j R x}}{N_{o}+P_{i} \cdot G_{i, j R x}}
\end{gathered}
$$

where $i$ represents the CUE, $j$ represents the D2D, $\gamma_{i, j}$ represents the SINR value of eNodeB, $\beta_{j T x, j R x}$ represents the SINR value of D2D, $G_{i, e N B}$ represents the channel gain of CUE to eNodeB, $G_{j T x, e N B}$ represents the channel gain of D2D TX to eNodeB, $G_{j T x}, j R x$ represents the channel gain of D2D TX to D2D RX, $G_{i, j R x}$ represents the channel gain of CUE to D2D RX, $P_{i}$ represents the transmit power of CUE, $P_{j}$ represents the transmit power of D2D and $N_{o}$ represents the noise. SINR in Equations 3 and 4 are loaded in the form of a matrix which will become the input for each allocation algorithm used.

\subsection{Problem Formulation}

This research aims to minimize the interference and obtain good performance from the D2D commu- 
nication system. This research uses a problem formulation defined on the equation below

$$
\begin{gathered}
\sum_{i=1}^{C} \mu c_{i, j}+\sum_{i=1}^{C} \sum_{j=1}^{D} \mu d_{i, j} \cdot x_{i, j}, \\
\sum_{i=1}^{C} x_{i, j} \leq 1, \forall j \in\{1, \ldots D\}, \\
\sum_{j=1}^{D} x_{i, j} \leq 1, \forall j \in\{1, \ldots C\}, \\
x_{i, j} \in\{0,1\}, \forall i \in\{1, \ldots n\} \operatorname{dan} \forall j\{1, \ldots m\} .
\end{gathered}
$$

where $\mu c_{i, j}$ on equation 5 represents the sum rate generated from the SINR of eNodeB and D2D. Equation 6 shows that a single CUE $i$ can only be paired with a single D2D, while Equation 7 shows that a single D2D $j$ can only use a single resource block of the CUE $i$. Equation 8 ensures that the variable of $x_{i, j}$ is a binary number 1 or $0 . x_{i, j}$ will value as 1 if the CUE shares its resources with the D2D pair. Otherwise, $x_{i, j}$ will be 0 .

\subsection{Proposed Algorithm}

\subsubsection{Greedy Algorithm}

The greedy algorithm is a resource scheduling algorithm in D2D communication, where it only allocates to the user with the best channel status. The order of the scheduling process is determined based on the order in which the user arrives. The user in the first order can choose the best channel to use. The allocated resource is forbidden to use for other D2D pairs. This allocation will continue until all the D2D pairs get resources from CUE [13].

\subsubsection{TAFIRA Algorithm}

The TAFIRA algorithm is a resource allocation algorithm in D2D communication. Based on the work [8] TAFIRA aims to minimize the total interference introduced in the system when the D2D pairs use the CUE channel while also ensure fairness in resource allocation. The algorithm uses the combined capacity of the eNodeB and D2D pair as an input parameter. If it cannot find any allocation in the first phase of the auction, then a second phase starts to find an allocation that satisfies the system sum-rate demand. This allocation will continue until all the D2D pairs get resources from CUE.

\subsection{Simulation Parameter}

The proposed TAFIRA D2D algorithm is analyzed and simulated with Python. The proposed algorithm compares with the greedy algorithm, and TAFIRA CUE then analyzes with parameters, namely spectral
Table 1: Simulation Parameter

\begin{tabular}{|c|c|}
\hline Parameters & Value \\
\hline Cell Radius & $500 \mathrm{~m}$ \\
\hline D2D Radius & $40 \mathrm{~m}$ \\
\hline Number of CUE & 50 \\
\hline Number of D2D pairs & $\begin{array}{c}\text { Between 5 to 50 } \\
\text { (multiples of 5) }\end{array}$ \\
\hline CUE Transmit Power & 0.5 watt \\
\hline D2D Transmit Power & 0.5 watt \\
\hline Number of Resource Block & 50 \\
\hline Resource Bandwidth & $180 \mathrm{KHz}$ \\
\hline Pathloss Model & UMi Pathloss \\
\hline Time Transmission Interval (TTI) & 1000 \\
\hline Noise & $-174 \mathrm{dbm} / \mathrm{Hz}$ \\
\hline
\end{tabular}

efficiency, power efficiency, and D2D fairness. Table 1 shows the simulation parameters of this research. Due to this research focuses on uplink communication, the transmit power for the CUE and D2D in this simulation will be equal.

\subsubsection{Spectral Efficiency}

Spectral efficiency explains how many bits per second (bps) that system can carry at a given frequency [14]. It will be more efficient if it has a high spectral efficiency value. Equation 5 calculates the spectral efficiency value. The enhancement of D2D users can improve the spectral efficiency performance in the system because the enhancement of D2D users can improve the sum-rate value. Thus it is also affecting the spectral efficiency of the system.

$$
\varepsilon_{i, j}=\frac{\sum_{i=0}^{I} \sum_{j=0}^{J} \rho_{i, j}}{B \cdot R B}
$$

where $\varepsilon_{i, j}$ represents the spectral efficiency, $\rho_{i, j}$ represents the sum-rate, and $R B$ represents the number of the resource block.

\subsubsection{Power Efficiency}

Power efficiency is the number of bits that can be sent from the transmitter every second in the total power allocated to the system [15]. Equation 6 calculates the power efficiency.

$$
\eta_{i, j}=\frac{\sum_{i=0}^{I} \sum_{j=0}^{J} \rho_{i, j}}{I \cdot \sum_{i=0}^{I} P_{i}+J \cdot \sum_{j=0}^{J} P_{j}}
$$

where $\eta_{i, j}$ represents the power efficiency, $I$ represents the number of CUE and $J$ represents the number D2D pair. 


\subsubsection{Fairness}

Fairness is the equal distribution of sum rates for each D2D pair obtained [16]. Equation 7 calculates the amount of fairness value.

$$
F I_{j T x, j R x}=\frac{\left(\sum_{i=0}^{I} \sum_{j=0}^{J} \rho_{i, j}\right)^{2}}{J \cdot \sum_{i=0}^{I} \sum_{j=0}^{J}\left(\rho_{i, j}\right)^{2}}
$$

\section{Results and Discussion}

\subsection{Performance Results of Spectral Efficiency}

Figure 3 shows the graph results of the spectral efficiency performance of each algorithm. It shows that the number of D2D users give an impact on spectral efficiency performance. The enhancement of D2D users can improve the spectral efficiency performance in the system because the enhancement of D2D users can improve the sum rate value. Thus it is also affecting the spectral efficiency of the system.

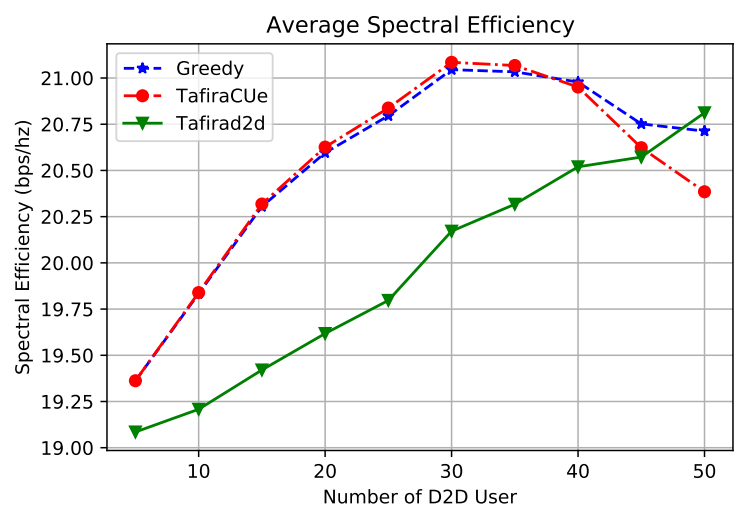

Fig. 3. Performance results of spectral efficiency.

Table 2 shows the comparison value of the spectral efficiency results of each algorithm. It shows that the higher spectral efficiency takes place in Greedy algorithm with the value $20.52 \mathrm{bps} / \mathrm{Hz}$. The result is $0.04 \mathrm{bps} / \mathrm{Hz}$ greater than the TAFIRA CUE algorithm and also $0.58 \mathrm{bps} / \mathrm{Hz}$ greater than the TAFIRA D2D algorithm. High spectral efficiency means that the Greedy algorithm has the best performance to transmit data every second in a hertz.

Table 2: Average Spectral Efficiency of Each Algorithm

\begin{tabular}{|c|c|}
\hline Algorithm & $\begin{array}{c}\text { Spectral Efficiency } \\
\text { (bps/Hz) }\end{array}$ \\
\hline Greedy & 20.52 \\
\hline TAFIRA CUE & 20.48 \\
\hline TAFIRA D2D & 19.94 \\
\hline
\end{tabular}

\subsection{Performance Results of Power Efficiency}

Figure 4 shows the graph results of the power efficiency performance of each algorithm. It shows that the number of D2D users give an impact on power efficiency performance. The enhancement of D2D users can affect the power efficiency performance in the system because it can decrease the power efficiency in the system. Thus, the power efficiency of the system tends to decrease.

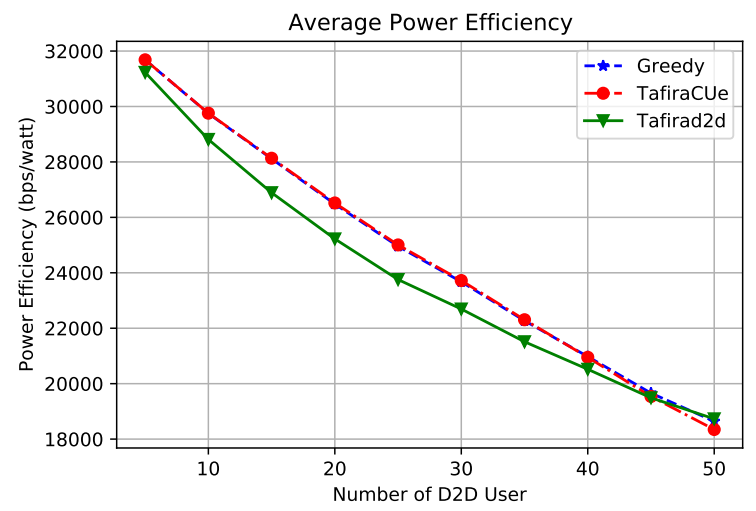

Fig. 4. Performance results of power efficiency.

Table 3 shows the comparison energy efficiency results of each algorithm. It shows that the higher power efficiency takes place in Greedy algorithm with a value of $24.62 \mathrm{Kbps} /$ watt. The result is $0.03 \mathrm{Kbps} /$ watt greater than the TAFIRA CUE algorithm and 0.74 Kbps/watt greater than the TAFIRA D2D algorithm. Thus it proves that the Greedy algorithm makes the power in the system more efficient.

Table 3: Average Power Efficiency of Each Algorithm

\begin{tabular}{|c|c|}
\hline Algorithm & $\begin{array}{c}\text { Power Efficiency } \\
\text { (Kbps/watt) }\end{array}$ \\
\hline Greedy & 24.62 \\
\hline TAFIRA CUE & 24.59 \\
\hline TAFIRA D2D & 23.88 \\
\hline
\end{tabular}

\subsection{Fairness Performance Results}

Figure 5 shows the graph results of the fairness performance of each algorithm. It shows that there is a reducing value of fairness number of D2D users hit 20 due to there is noise. But as increase the number of D2D users then it also affects the instability value of fairness. It is due to the value of fairness number of D2D users depends on the value of the sum rate and the number of D2D pairs.

Table 4 shows the comparison of the fairness results of each algorithm. It shows that the higher fairness is in the Greedy algorithm with the value of 0.91 , 


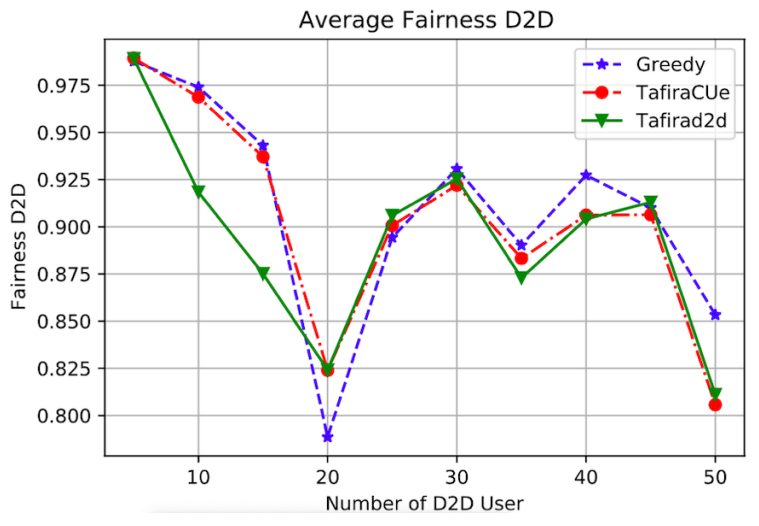

Fig. 5. Performance results of fairness.

which is 0.01 greater than the TAFIRA CUE algorithm also 0.02 greater than the TAFIRA D2D algorithm. The value of it is still not quite good, due to there is still lack of ability dividing all resources fairly.

Table 4: Fairness of Each Algorithm

\begin{tabular}{|c|c|}
\hline Algorithm & Fairness \\
\hline Greedy & 0.91 \\
\hline TAFIRA CUE & 0.9 \\
\hline TAFIRA D2D & 0.89 \\
\hline
\end{tabular}

\section{Conclusion}

The research discusses performance simulation comparison of the resource allocation scheme between the TAFIRA algorithm and the conventional Greedy algorithm. The simulation uses the number variations of the D2D pairs in the system. The results show that the TAFIRA D2D algorithm obtains a lower performance than the Greedy Algorithm with value results of $19.94 \mathrm{bps} / \mathrm{Hz}$ in spectral efficiency, $23.88 \mathrm{Kbps} /$ watt in power efficiency, and 0.89 in the fairness among D2D pairs. It is due to the TAFIRA algorithm has two-phase where all of the large allocation resources have been allocated for the D2D pair in the first phase, which can cause a small allocation resource for the D2D pair who got the resource from the second phase. In addition, adding a power control in every algorithm might resolve the instability result of performance. Thus, it can be an opportunity for future works.

\section{References}

[1] C. B. Das, A Study on Device To Device Communication in Wireless Mobile Network, Int. J. Mod. Commun. Technol. Res., vol. 3, no. 3, pp. $15,2015$.

[2] S. T. Shah, J. Gu, S. F. Hasan, and M. Y. Chung, SC-FDMA-based resource allocation and power control scheme for D2D communication using LTE-A uplink resource, Eurasip J. Wirel. Commun. Netw., vol. 2015, no. 1, 2015.

[3] D. Della Penda, L. Fu, M. Johansson, Mode selection for energy efficient D2D communications in dynamic TDD systems, in: Proc. IEEE Int. Conf. on Commun. (ICC), London, United Kingdom, 2015, pp. 54045409.

[4] A. Asadi, Q. Wang, and V. Mancuso, A Survey on Device-to-Device Communication in Cellular Networks, IEEE Communications Surveys Tutorials, vol. 16, no. 4, pp. 18011819, 2014.

[5] C. Ma, J. Liu, X. Tian,H. Yu, Y. Cui and X. Wang, Interference exploitation in D2D-enabled cellular networks: A secrecy perspective, IEEE Trans. Commun., vol. 63, no. 1, pp. 229-242, Jan. 2015.

[6] S. Selmi and R. Bouallegue, Interference and power management algorithm for D2D communications underlay $5 \mathrm{G}$ cellular network, Int. Conf. Wirel. Mob. Comput. Netw. Commun., vol. 2019October, pp. 18, 2019.

[7] X. Li, R. Shankaran, M. A. Orgun, G. Fang, and Y. Xu, Resource Allocation for Underlay D2D Communication with Proportional Fairness, IEEE Trans. Veh. Technol., vol. 67, no. 7, pp. 62446258, 2018.

[8] M. T. Islam, A. E. M. Taha, S. Akl, and S. Choudhury, A two-phase auction-based fair resource allocation for underlaying D2D communications, 2016 IEEE Int. Conf. Commun. ICC 2016, 2016.

[9] M. Y. Hassan, F. Hussain, M. S. Hossen, S. Choudhury, and M. M. Alam, A near optimal interference minimization resource allocation algorithm for D2D communication, IEEE Int. Conf. Commun., 2017.

[10] ITU-R, M.2135 Guidelines for evaluation of radio interface technologies for IMT-Advanced, 2009.

[11] M. I. Khan, M. M. Alam, Y. Le Moullec, and E. Yaacoub, Cooperative reinforcement learning for adaptive power allocation in device-todevice communication, IEEE World Forum Internet Things, WF-IoT 2018 - Proc., vol. 2018January, pp. 476481, 2018.

[12] Song, Xin, et al. Joint uplink and downlink resource allocation for D2D communications system. Future Internet 11.1 (2019): 12. 
[13] V. S. W. Prabowo, B. Pamukti, A. Fahmi, N. M. Adriansyah, and N. Andini, Joint-greedy allocation algorithm on D2D communication underlaying networks, Proc. - 2019 IEEE Asia Pacific Conf. Wirel. Mobile, APWiMob 2019, pp. 4852, 2019.

[14] J. Iqbal, M. A. Iqbal, A. Ahmad, M. Khan, A. Qamar, and K. Han, Compa- rison of Spectral Efficiency Techniques in Device-to-Device Communication for 5G, IEEE Access, vol. 7, pp. 57 44057 449, 2019.

[15] H. Xu, W. Xu, Z. Yang, Y. Pan, J. Shi, and M. Chen, Energy-Efficient Resource Allocation in D2D Underlaid Cellular Uplinks, IEEE Commun. Lett., vol. 21, no. 3, pp. 560563, 2017.

[16] Jain, Rajendra K., Dah-Ming W. Chiu, andWilliam R. Hawe. A quantitative measure of fairness and discrimination. Eastern Research Laboratory, Digital Equipment Corporation, Hudson,MA (1984).

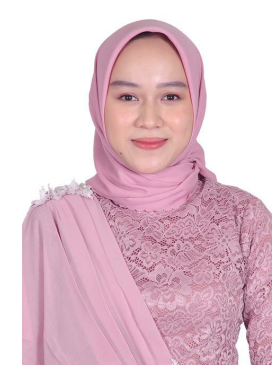

Alfiya Syifana was born in Tasikmalaya, Indonesia, in 1999. Alfiya is pursuing a Bachelors Degree in Telecommunication Engineering at the school of Electrical Engineering, Telkom University. She finished her Bachelor degree at 2021. Her reasearch interest include wireless communication and $4 \mathrm{G}$ developments.

Linda Meylani received the B.Eng degree from STT Telkom Bandung in 2001, received M.Eng degree from STT Telkom Bandung in 2006 and received the D.Eng degree from Bandung Institute of Technology in 2021, respectively. Currently, she is a lecturer at School of Electrical Engineering, Telkom University,

Bandung. Her research interest is multiple access scheme and wireless communication.

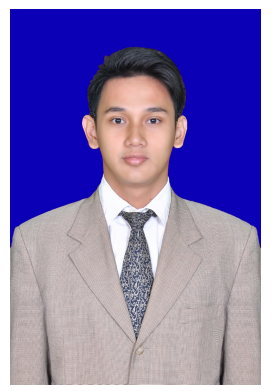

\section{Vinsensius Sigit Widhi}

Prabowo was born in Jakarta (Indonesia), in 1993. He received the B.S. degree in Telecommunication engineering from Telkom University, Bandung, Indonesia, in 2015, M.S. degree in Electrical engineering from the Telkom University, Bandung, Indonesia, in 2017.

Since 2016, he is Tenured Lecturer with the School of Electrical Engineering, Telkom University. He is the author of Pengalokasian Sumber Daya Radio pada Sistem Komunikasi Pita Lebar, and more than 20 indexed articles. His research interests include wireless communication system, radio resources management, and Telecommunication Transmission. 